\title{
A Study on Identification and Antifungal Susceptibility Pattern of different Candida Species Isolated from Various Clinical Specimens in a Tertiary care Hospital of West Bengal, India
}

\author{
Indrajit Gupta $^{1 *}$, Purbasha Ghosh ${ }^{1}$, Ayan Nandi ${ }^{1}$, Prosenjit Naskar ${ }^{2}$, \\ Sayantan Mondal ${ }^{1}$ and G.D. Mitra ${ }^{1}$ \\ ${ }^{1}$ Department of Microbiology, Burdwan Medical College, West Bengal, India \\ ${ }^{2}$ Department of Community Medicine, Burdwan Medical College, West Bengal, India \\ *Corresponding author
}

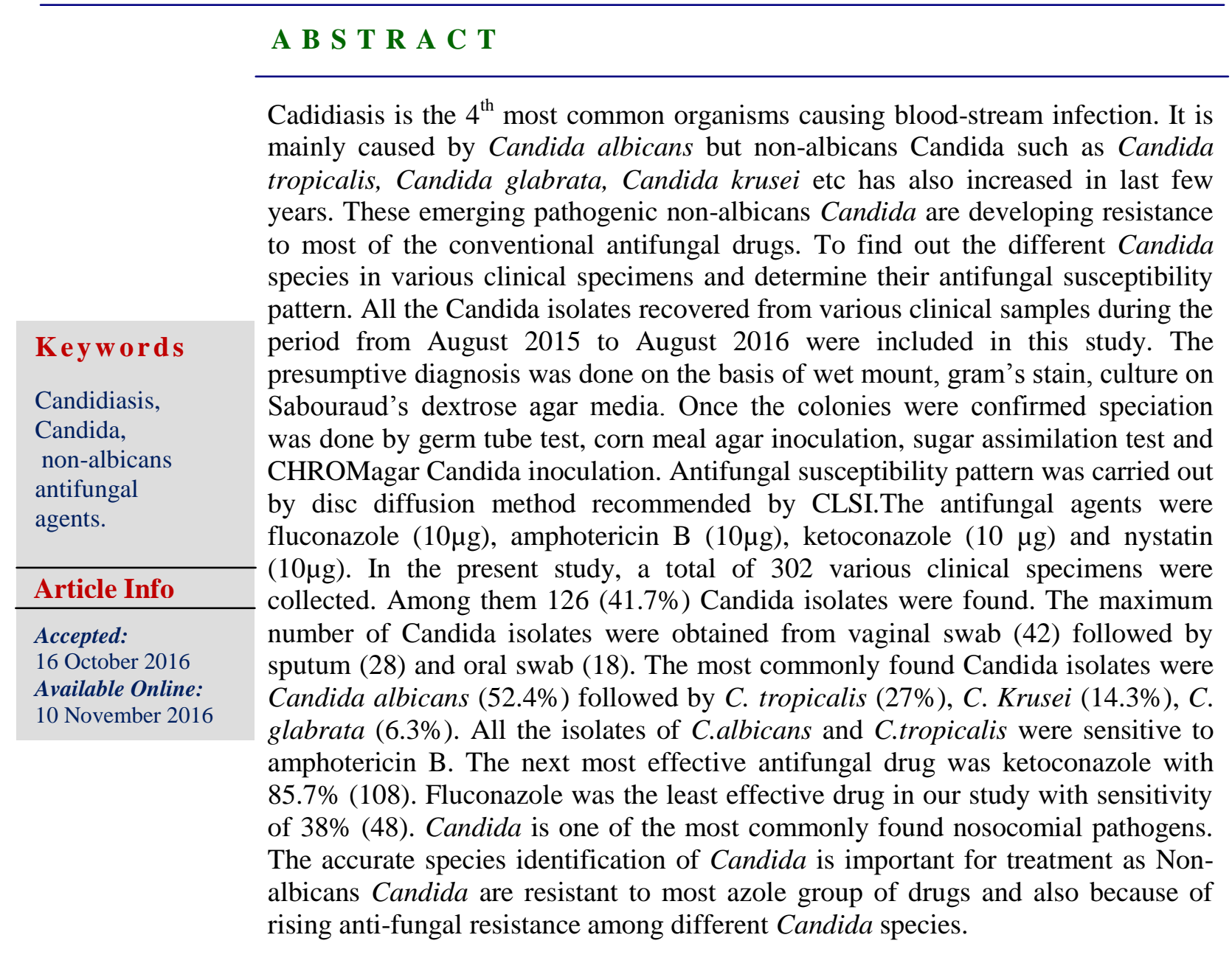




\section{Introduction}

Candida is yeast like fungus which causes commonest fungal disease (called Candidiasis) in humans affecting skin, mucosa, nails and various internal organs. It is caused by various species belonging to genus Candidia with Candida albicans as the representative species (Chander, 2009). However in recent years non-albicans Candida are increasingly found in various clinical scenarios (Gullo, 2009). The infection may be acute or chronic, superficial or deep and its clinical spectrum is wide. It is found mainly as secondary infection individuals with some underlying immunocompromised conditions such as prolonged usage of antibiotics, chemotherapy, immunosuppressive drugs, long term catheterization etc.

Species identification of Candida is very much important especially for treatment purposes as not all species respond to the same treatment because of the development of antifungal resistance (Shivanand et al., 2011). So the objective of this present study is Candida species identification among various clinical specimens and to determine their antifungal drug susceptibility.

\section{Materials and Methods}

The present study was conducted in the department of Microbiology, in a peripheral tertiary medical college of west Bengal for a period of one year i.e. from August 2015 to August 2016.All the clinical samples from those patients having suspected fungal infections submitted in the microbiology department were included in this study. Samples were collected from blood, urine, sputum, oral swab, vaginal swab, pus, skin scrapping and nail scrapping. The preliminary presumptive diagnosis was made on the basis of wet mount, gram's stain, culture on SDA (Sabouraud's dextrose agar) media. (Figure-1) Isolates other than Candida species were excluded from the study. Colonies were identified and confirmed by colony character and Gram's stain. Once the colonies were confirmed Candida species identification was done by germ tube test, corn meal agar inoculation, sugar assimilation test and CHROMagar Candida inoculation. Antifungal drug susceptibility testing was done by disc diffusion method on Muller Hinton Agar recommended by CLSI (CLSI, 2016). The antifungal discs used were fluconazole $(10 \mu \mathrm{g}), \quad$ amphotericin B $(10 \mu \mathrm{g})$, ketoconazole $(10 \mu \mathrm{g})$ and nystatin $(10 \mu \mathrm{g})$.

The study proposal along with other relevant documents was submitted to the 'Institutional Ethics Committee' for review and approval. The study was commenced only after approval is obtained from appropriate authority.

\section{Statistical Analysis}

Data were coded and entered into MS-Excel sheet. Statistical analysis were done using software SPSS 20 version. Descriptive and inferential statistics were used. Data were presented in percentages.

\section{Results and Discussion}

In the present study, a total of 302 various clinical specimens were collected. Among them $126(41.7 \%)$ Candida isolates were found. The maximum number of Candida isolates were obtained from vaginal swab (42) followed by sputum (28) and oral swab (18).This distribution is displayed in Table1.

The most commonly found Candida isolates were Candida albicans (66) followed by $C$. tropicalis (34), C. Krusei (18), C. glabrata (08).The percentage wise distribution of different Candida species is shown in pie diagram-2. 
All the isolates of C.albicans and C.tropicalis were sensitive to amphotericin B. The next most effective antifungal drug was ketoconazole with $85.7 \%$ (108). Fluconazole was the least effective drug in our study with sensitivity of $38 \%$ (48). Antifungal sensitivity pattern of Candida isolates is shown in Table-2.

The frequency of diseases caused by Candida has amplified over the last few years especially in immunocompromised patients. There is also an increased frequency of non-albicans candida commonly Candida tropicalis, Candida glabrata, Candida krusei. Our study shows occurrence of Candida infection was $41.7 \%$ in various clinical specimens. This finding is similar with the study done by Mohandas et al., (2011).

In our present study, highest numbers of isolates $(52.40 \%)$ were Candida albicans followed by C. tropicalis (27\%), C. krusei $(14.30 \%)$ and $C$. glabrata $(6.30 \%)$. These findings also correlates with Mohandas et al., (2011) study.

In this study, speciation of Candida was done by germ tube test, corn meal agar inoculation, sugar assimilation test and CHROMagar Candida inoculation. Chromogenic agar media is a differential medium used in mycology laboratory for rapid identification of Candida spp. in comparison to conventional methods. It contains enzymatic substrates linked to different chromogenic compounds. When a specific substrate breaks down the substrate, the chromogenic substrate produce colours. The different colour variation is useful for the rapid and presumptive identification of Candida (Odds et al., 1994). Consequently this will help for the early initiation of appropriate therapy.

Table.1 Distribution of Candida isolates according to various clinical specimens:

\begin{tabular}{|c|c|c|}
\hline Specimen & Number of isolates & Percentage \\
\hline Vaginal swab & 42 & $33.3 \%$ \\
\hline Sputum & 28 & $22.2 \%$ \\
\hline Oral swab & 18 & $14.3 \%$ \\
\hline Blood & 06 & $4.8 \%$ \\
\hline Pus \& wound swab & 08 & $6.3 \%$ \\
\hline Urine & 12 & $9.5 \%$ \\
\hline Nail scraping & 05 & $4.0 \%$ \\
\hline Skin scrapings & 07 & $5.6 \%$ \\
\hline
\end{tabular}

Table.2 Antifungal sensitivity pattern of Candida isolates

\begin{tabular}{|c|c|c|c|c|}
\hline & \multicolumn{4}{|c|}{ Candida species } \\
\hline $\begin{array}{c}\text { Antifungal } \\
\text { agents }\end{array}$ & $\begin{array}{c}\text { C. albicans } \\
(\mathrm{n}=66)\end{array}$ & $\begin{array}{c}\text { C. tropicalis } \\
(\mathrm{n}=34)\end{array}$ & $\begin{array}{c}\text { C. } \text { krusei } \\
(\mathrm{n}=18)\end{array}$ & $\begin{array}{c}\text { C. glabrata } \\
(\mathrm{n}=08)\end{array}$ \\
\hline Amphotericin B & $100 \%$ & $100 \%$ & $96.7 \%$ & $98.1 \%$ \\
\hline Nystatin & $94 \%$ & $92.5 \%$ & $96.3 \%$ & $100 \%$ \\
\hline Fluconazole & $62.7 \%$ & $66 \%$ & $28.4 \%$ & $\mathrm{R}$ \\
\hline ketoconazole & $92.3 \%$ & $79 \%$ & $69.7 \%$ & $49.2 \%$ \\
\hline
\end{tabular}


Fig.1 Growth of Candida sp. in Sabouraud's Dextrose agar:

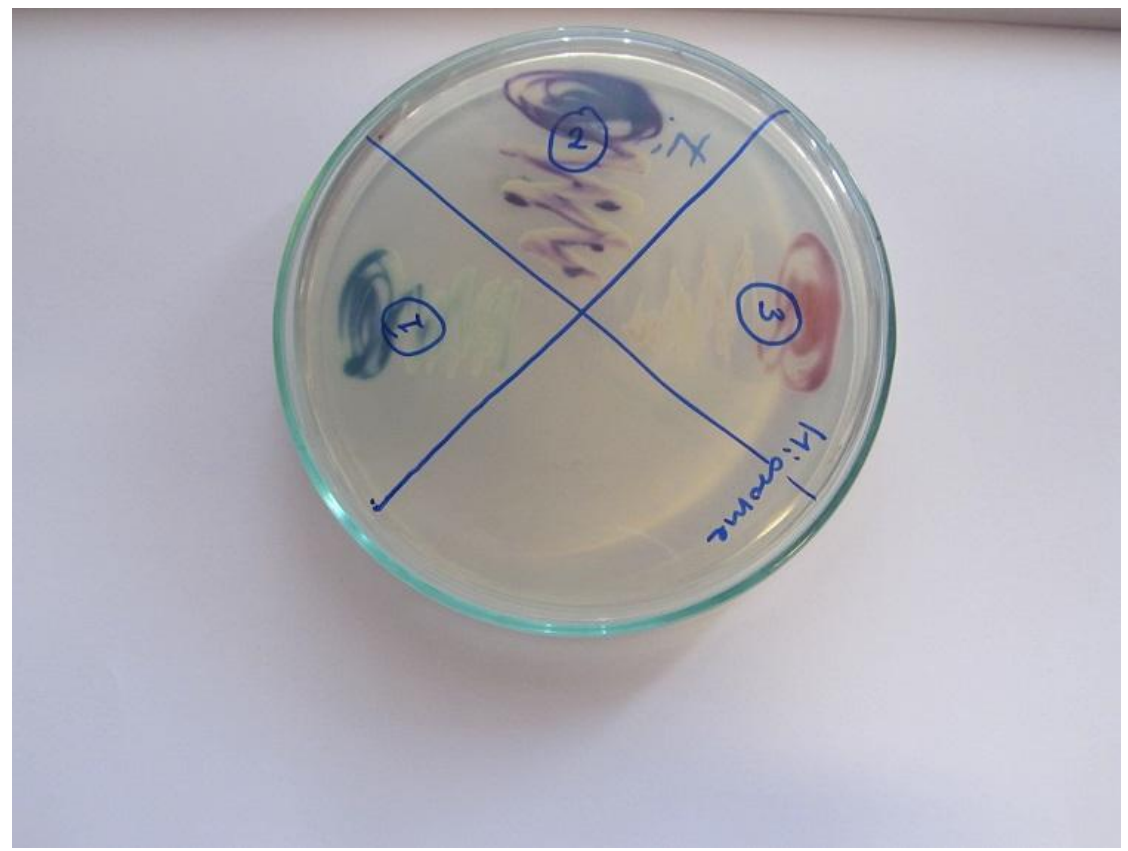

Fig.2 Pie diagram showing distribution of different Candida species:

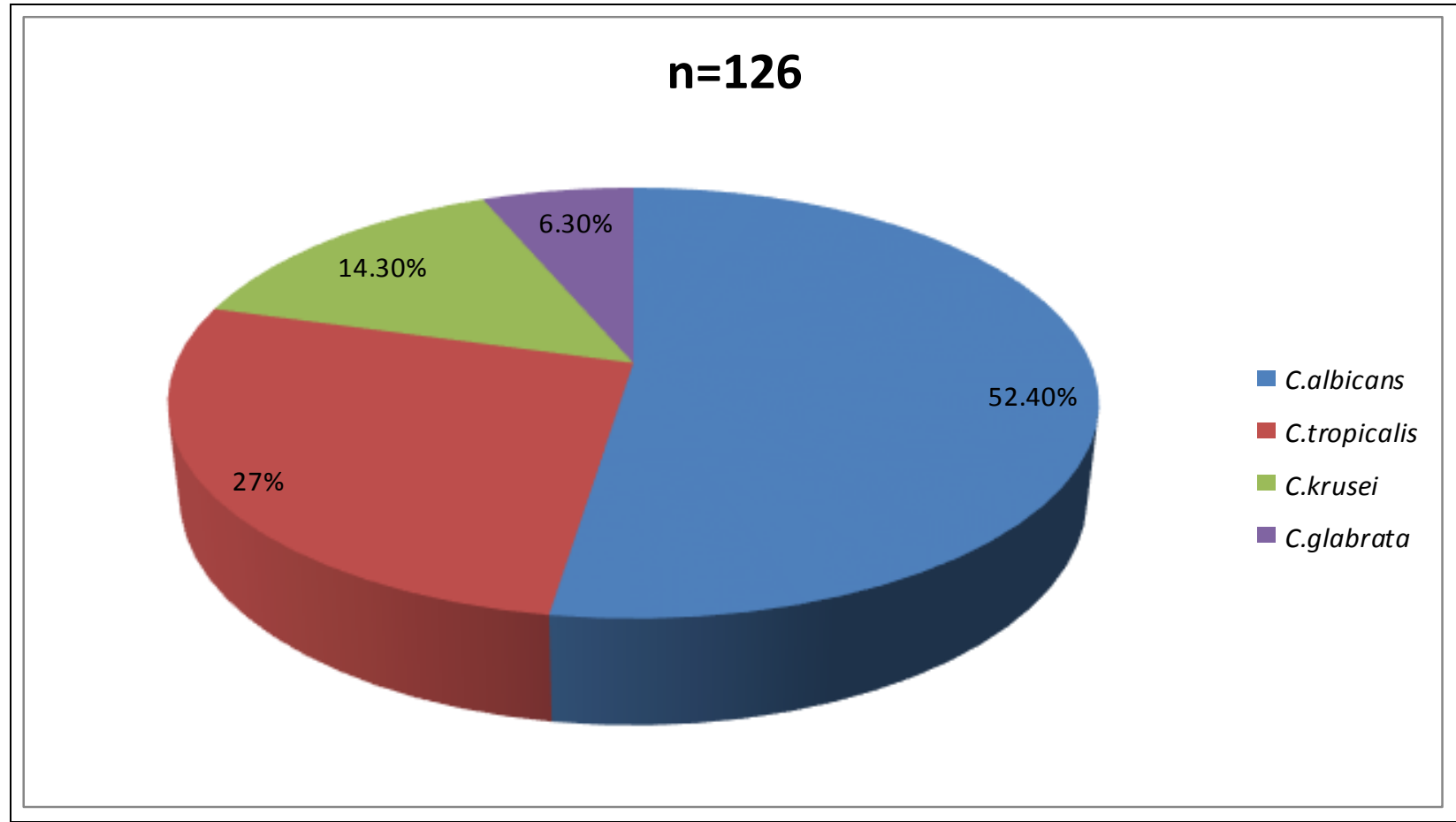

Our study showed that Amphotericin B was the most effective antifungal drug. This finding is similar with another study done by Maria Fatima Sugizaki et al., (1998). The next most effective antifungal drug was ketoconazole with $85.7 \%$ (108). Fluconazole was the least effective drug in our study with sensitivity of $38 \%$ (48). These findings 
correlate with study done by Ananth kashid et al., (2011).

In conclusion, Candida is one of the most commonly found nosocomial pathogen. The accurate species identification of Candida is important for treatment as Non-albicans Candida are resistant to most azole group of drugs and also because of rising anti-fungal resistance among different Candida species.

This study therefore emphasizes the need for rapid and precise identification of different Candida isolates to species level for effective treatment and management strategies. The present study also advocates the need for periodic surveillance of the antifungal drug susceptibility pattern of the prevalent various Candida species, as it would enlighten the judicious use of antifungal therapy in patients.

\section{Acknowledgement}

We express our sincere thanks to Professor Sukumar Basak, Principle of Burdwan Medical College for his kind support and allowing us to conduct this study.

\section{References}

Ananth Kashid, Sandhya Belawadi, Gaytri Devi, Indumal. 2011. Characterisation and antifungal susceptibility testing for Candida in a tertiary care hospital. J. Health Sci. Res., 2(2): 1-1.

Chander, J. 2009. Textbook of Medical
Mycology. $3^{\text {rd }}$ edition, Chapter-20, Candidiasis, p-266.

Clinical Laboratory Standards Institute (CLSI). 2016. Method for antifungal disk diffusion susceptibility testing of yeasts. Approved guidelines. $26^{\text {th }}$ edition, CLSI document - M44 - A2.

Gullo, A. 2009. Invasive fungal infections: the challenge continues. Drugs, 69: 65-73.

Maria Fátima Sugizaki, Cristianne Roberta Rhoden, Denise Mara Bombonatti. 1998. Prevalence and in vitro antifungal susceptibility of Candida spp. isolated from clinical specimens in São Paulo, Brazil. Rev Iberoam Micol., 15: 16-18.

Mohandas, V., Ballal, M. 2011. Distribution of Candida species in different clinical samples and their virulence: biofilm formation, proteinase and phospholipase production: a study on hospitalized patients in Southern India. J. Global Infect. Dis., 3(1): 4-8. doi:10.4103/0974-777X.77288.

Odds, F.C., Bernaerts, R. 1994. CHROM agar Candida, a new differential isolation medium for presumptive identification of clinically important Candida spp. J. Clin. Microbiol., 32(8): 1923-9.

Shivanand Dharwad, Saldanha Dominic, R.M. 2011. Species identification of candida isolates in various clinical specimens with their anti-fungal susceptibility patterns. J. Clin. Diag. Res., 5(6): 1177-1181.

\section{How to cite this article:}

Indrajit Gupta, Purbasha Ghosh, Ayan Nandi, Prosenjit Naskar, Sayantan Mondal and G.D. Mitra. 2016. A Study on Identification and Antifungal Susceptibility Pattern of different Candida Species Isolated from Various Clinical Specimens in a Tertiary care Hospital of West Bengal. Int.J.Curr.Microbiol.App.Sci. 5(11): 346-350. doi: http://dx.doi.org/10.20546/ijcmas.2016.511.039 